\title{
Negative Cycle Detection Problem
}

\author{
Chi-Him Wong and Yiu-Cheong Tam \\ The Chinese University of Hong Kong \\ \{02658924, 02654543\}@alumni.cse.cuhk.edu.hk
}

\begin{abstract}
In this paper, we will describe some heuristics that can be used to improve the runtime of a wide range of commonly used algorithms for the negative cycle detection problem significantly, such as Bellman-Ford-Tarjan (BFCT) algorithm, Goldberg-Radzik (GORC) algorithm and Bellman-Ford-Moore algorithm with Predecessor Array (BFCF). The heuristics are very easy to be implemented and only require modifications of several lines of code of the original algorithms. We observed that the modified algorithms outperformed the original ones, particularly in random graphs and no cycle graphs. We discovered that $69 \%$ of test cases have improved. Also, the improvements are sometimes dramatic, which have an improvement of a factor of 23 , excluding the infinity case, while the worst case has only decreased by $85 \%$ only, which is comparably small when compared to the improvement.
\end{abstract}

\section{The Negative Cycle Detection Problem}

\subsection{Introduction}

The Negative Cycle Detection problem has numerous applications in model verification, compiler construction, software engineering, VLSI design, scheduling, circuit production, constraint programming and image processing. For example, Constraint-based program analysis requires feasibility checking of constraint sets. Constraint graphs are often used to represent systems of difference constraints; an application of Farkas' Lemma shows that a system of difference constraints is feasible if and only if there are no negative cost cycles in the corresponding constraint graph. That is, a difference constraint problem is feasible if and only if there are no negative cycles in the graph.

In the design of VLSI circuits, it is required to isolate negative feedback loops. These negative feedback loops correspond to negative cost cycles in the amplifiergain graph of the circuit. The problem of checking whether a zero-clairvoyant scheduling system has a valid schedule can also be reduced to the problem of identifying negative cost cycles in the appropriate graph. Recent approaches to the image segmentation problem are also based on negative cycle detection. Most of the approaches are based on the famous Bellman-Ford (BF) algorithm. All

\footnotetext{
${ }^{0}$ The work described in this paper was partially supported by a direct allocation grant from the Research Grant Council of the Hong Kong Special Administrative Region, China (Project No. 2050321).
} 
these algorithms have their worst case bound of $O(|V||E|)$ in runtime. Most of the previous algorithms run relatively slow on no cycle graphs. However, no cycle graph is common in practice. So we try to develop some heuristics that are good in detecting no cycle graphs. Our heuristics can be used to improve the runtime of a wide range of Bellman-Ford based algorithms. We discovered that $69 \%$ of test cases have improved. Also, the improvements are sometimes dramatic, which have an improvement of a factor of 23 , excluding the infinity case, while the worst case has only decreased by $85 \%$ only, which is comparably small when compared to the improvement. There are also significant improvement in no cycle graphs and random graphs.

\section{Related Works}

\subsection{Definitions}

The Negative Cycle Detection problem can be defined as the problem of deciding whether a negative cost cycle exists in a directed graph. This does not require finding a path from a particular source to a particular destination, as it is only a decision problem and require only a yes or no answer. Formally, the Negative Cycle Detection (NCD) problem is defined as follows:

Given a directed graph $G=\langle V, E, c\rangle$, where $V=\left\{v_{0}, v_{1}, \ldots, v_{n-1}\right\}$, $|V|=n, E=\left\{e_{i j}: v_{i} \rightarrow v_{j}\right\},|E|=m$, and a cost function $c: E \rightarrow Z$, is there a negative cost cycle in $G$ ?

There are no restrictions on the edge costs, i.e., they can be arbitrary integers as opposed to small integers, as required by some scaling algorithms. We can even extend the weights to floating point numbers.

Our heuristics can be applied to a wide range of commonly used algorithms for the Negative Cycle Detection problem that is a single source algorithm. In the following, we will define the meaning of single source algorithm.

An algorithm for the negative cycle detection problem is defined as a single source algorithm if and only if the algorithm is relaxation-based and all the relaxations (or calculations of labels) originate from a single vertex (the source).

We will give some examples of single source algorithms in the following.

\subsection{The Bellman-Ford Moore Algorithm with Predecessor Array (BFCF)}

The Bellman-Ford-Moore (BFFI) algorithm attempts to reduce the number of vertices that must be examined in each stage of the "standard" Bellman-Ford (BF) algorithm by using a First-In-First-Out (FIFO) queue to store the vertices whose distance labels were changed in the previous stage.

Predecessor Array is a strategy that uses parent pointer to store the parent of each vertex $v_{i}$, where the parent of $v_{i}$ is the vertex that caused the most recent label change to $v_{i}$. This strategy is widely used in many Bellman-Ford based algorithms. 
The Bellman-Ford-Moore algorithm with Predecessor Array (BFCF) is an algorithm that combines the above two techniques. As the algorithm starts with a vertex with label 0 and all the other vertices of label infinity, it is a single source algorithm. Notice that BFCF still has the worst case bound of $O(|V||E|)$ in runtime.

\subsection{The Bellman-Ford-Tarjan Algorithm (BFCT)}

Another variation of the BF algorithm is BFCT, which combines the "standard" $\mathrm{BF}$ algorithm with the FIFO queue of BFFI and the sub-tree disassembly cycle detection strategy due to Tarjan. The sub-tree disassembly strategy is implemented by describing the tree structure by, besides the usual predecessor function, the first son and the adjacent brother function (next and previous). This allows traversals of sub-trees in linear time, and tree modifications in constant time. When no cycle is found in the traversal of a sub-tree $T_{v}$, all vertices in $T_{v}$ will be discarded from the queue and the tree. [Tar81]. A negative cycle will be detected when a tree path from a vertex goes back to one of its ancestors. As the algorithm is started with one source of label 0 and all the other vertices of labels infinity, it is a single source algorithm.

\subsection{The Goldberg-Radzik Algorithm (GORC)}

The Goldberg-Radzik algorithm improved the Bellman-Ford-Moore algorithm. It achieves the same worst-case bound of $O(|V||E|)$, but can usually outperform BFFI in practice. The algorithm maintains the set of labelled vertices in two queues, queue $A$ and queue $B$. Vertices in queue $A$ will undergo a BellmanFord-Moore pass while vertices in queue $B$ will undergo a depth first search with no update. At the beginning of the algorithm, the source is put in queue $B$. In pass $B$, if there is an outgoing arc with reduced cost including zero, the path is traversed and all vertices visited will be put in queue $A$. Notice that there are no updates on the vertices' labels in this step. Also, if a reduced path from a vertex goes back to one of its ancestors, a negative cycle is detected. On the other hand, pass $A$ is a one step Bellman-Ford-Moore pass that relaxes the vertices in queue $A$ following the queue order. Notice that topological sort will be done after the depth first searches in pass $B$, so we will relax the vertices in pass $A$ topologically and the label will be updated.

The algorithm starts with one source of label 0 and all the other vertices of labels infinity, it is a single source algorithm.

\section{Our New Approach to This Problem}

Our approach is to incorporate two heuristics to the above single source algorithms.

\subsection{Heuristic One: Pumping Negative Strategy}

The first heuristic is based on an observation that in any negative cost cycle, we can find a negative weighted edge $e$ such that when we travel through the 
negative cost cycle starting from $e$, the accumulated costs are always negative. The formal definition of this lemma is as follows.

Lemma 1: Given a directed graph $G=\langle V, E, c\rangle$, where $V=\left\{v_{0}, v_{1}, \ldots, v_{n-1}\right.$ \}$,|V|=n, E=\left\{e_{i j}: v_{i} \rightarrow v_{j}\right\},|E|=m$, and $c$ is cost function $E \rightarrow Z$, with a negative cost cycle $\left\{v_{\pi(1)}, v_{\pi(2)}, v_{\pi(3)}, \ldots, v_{\pi(k)}\right\}$, where $v_{\pi(1)}=v_{\pi(k)}$, there exists at least one node $v_{\pi(i)}$ where $1 \leqq i \leqq k$ such that when we travel through the negative cost cycle starting from $v_{\pi(i)}$, the accumulated costs are always negative.

In this heuristic, we will stop the relaxation once the label becomes positive. But since we do not know which vertex in the negative cycle is the starting vertex with the property as stated in Lemma 1, we need to treat each vertex as source once. The proof of Lemma 1 is shown in Appendix II.

\subsection{Heuristic Two: Reduced Cost Elimination}

This heuristic is simply not deleting the labels on the vertices when moving from one pass to another with different vertices as the source. Consider a case where a particular vertex relaxes another vertex with label that is not marked 0 . If the label is smaller than the accumulated cost, we will have the following two conclusions:

1. That particular vertex has been travelled previously as it has a label that is not 0 .

2. The accumulated cost of the previous travel must be smaller than current accumulated cost as the label is smaller than the current accumulated cost.

Therefore, as the current accumulated cost is larger, using the current accumulated cost will reduce the number of vertices visited. Therefore, we can keep the cost label.

\section{Modifications on Various Algorithms}

In this section, we will briefly describe how we implement our heuristics into some single source algorithms.

\subsection{Modification of BFCF}

For heuristic one, we first change the algorithm such that the original BFCF algorithm will run $V$ times with a different vertex as the source each time. We also add a condition for the relaxation process which is, a relaxation can only be done and continued if the accumulated cost is negative. When there are no more relaxations, a new source will be chosen and the whole process is repeated. For heuristic two, we can implement it simply by keeping the cost label on each vertex unchanged when we start a new pass with a new source. The pseudo-code is as follows:

Initialize all cost labels to $0 .{ }^{*}$ So, all accumulated cost will be negative (Heuristic 1)*/ 
For all $\mathrm{v} / *(\text { Heuristic } 1)^{*} /$

\{

$\mathrm{BFCF}$ with $\mathrm{v}$ as the source $/ *(\text { Heuristic } 1)^{*} /$

$/ *$ Keep all the labels unchanged (Heuristic 2)*/

\}

\subsection{Modifications of BFCT}

The modifications to BFCT are similar to that in BFCF. However, each vertex in BFCT will be involved in the first son and adjacent brother functions (next and previous). During our modification, we can leave the values of the function (next and previous) unchanged when we choose a new starting point. This will improve the runtime without affecting the correctness.

\subsection{Modifications of GORC}

The modifications for GORC are a bit different from that of BFCF and BFCT. For heuristic one, we need to modify the condition in pass $B$ to ensure that the depth first search traversal will only traverse an edge when it leads to a negative reduced cost. GORC has already implemented the reduced cost elimination heuristic, so we do not need to do any modification for heuristic two.

\section{Experimental Setups}

\subsection{Results}

The problem generator was developed by the authors of [Gol95]. It can be downloaded from the website http://www.avglab.com/andrew/index.html. There are several sets of data.

1. The Rand-5 families have a fixed network size $n=2000000$ and $m=10000000$. The maximum arc length $U$ is fixed at 32000 and the minimum arc length $L$ varies from 0 to -64000 [Gol95].

2. The SQNC families represent the square grid families. Vertices of these networks correspond to points on the $x-y$ plane with integer coordinates $[x$, $y], 0 \leqq x \leqq X, 0 \leqq y \leqq Y$ and $X=Y$. The SQNC01 family has no cycles. The SQNC02 family has sparse small negative cycles. The SQNC03 family has dense small negative cycles. The SQNC04 family has several long cycles. The SQNC05 family has Hamilton cycle.

3. The LNC families are the grid networks mentioned above but with $Y=16$. The LNC01, LNC02, LNC03, LNC04 and LNC05 are similar to those in the SQNC families.

4. The PNC families are the layered networks. A layered network consists of layers $0 \ldots X-1$. Each layer is a simple cycle plus a collection of arcs connecting randomly selected pairs of vertices on the cycle. In the PNC families each layer contains 32 vertices and $X=n / 32$ [Gol95]. The PNC01, PNC02, PNC03, PNC04 and PNC05 are similar to those in the SQNC families. 
There are 5 test cases for each row shown in the following tables. The number of scan operations shown is the average of the 5 test cases. The percentage improvement is calculated according to the number of operation of relaxing. Results with more than $10 \%$ faster or slower will be highlighted in different colors. Finally, the 'M' in front of the name of the algorithm implies the modified algorithm with our heuristics implemented. The results are shown in Appendix I.

\section{Conclusion}

In this paper, we described two heuristics that can be incorporated into a wide range of commonly used single source algorithms for the Negative Cycle Detection problem. The modifications are very simple, involving only adding several lines of code.

After modification, all algorithms have increased the speed generally. There are significant results in the set of random graphs and no cycle graphs. We discovered that $69 \%$ of test cases have improved. Also, the improvements are sometimes dramatic, which have an improvement of a factor of 23, excluding the infinity case, while the worst case has only decreased by $85 \%$ only, which is comparably small when compared to the improvement.

\section{References}

[CG96] Boris V. Cherkassky and Andrew V. Goldberg. Negative-cycle detection algorithms. In Josep D'ıaz and Maria Serna, editors, Algorithms-ESA '96, Fourth Annual European Symposium, volume 1136 of Lecture Notes in Computer Science, pages 349-363, Barcelona, Spain, 25-27 September 1996. Springer.

[Gol95] Andrew V. Goldberg. Scaling algorithms for the shortest paths problem. SIAM Journal on Computing, 24(3):494-504, June 1995.

[Tar81] R. E. Tarjan. Shortest Paths. Technical report, AT\&T Bell Laboratories, Murray Hill, NJ, 1981. 


\section{Appendix I}

Table 1. Results for no cycle graphs LNC01

\begin{tabular}{|c||c|c|c|c||c|c|c|}
\hline & \#Vertices & MGORC & GORC & Improve & MBFCT & BFCT & Improve \\
\hline LNC01 & 8193 & 29571 & 51894 & $75.5 \%$ & 14891 & 27338 & $83.6 \%$ \\
\hline & 16385 & 59399 & 102501 & $72.6 \%$ & 29864 & 54115 & $81.2 \%$ \\
\hline & 32769 & 117508 & 204668 & $74.2 \%$ & 59016 & 107378 & $81.9 \%$ \\
\hline & 65537 & 236006 & 412421 & $74.8 \%$ & 118558 & 217019 & $83.0 \%$ \\
\hline & 131073 & 471187 & 824874 & $75.1 \%$ & 236706 & 433352 & $83.1 \%$ \\
\hline & 262145 & 940478 & 1643797 & $74.8 \%$ & 472542 & 864805 & $83.0 \%$ \\
\hline & 524289 & 1881286 & 3289308 & $74.8 \%$ & 945099 & 1733480 & $83.4 \%$ \\
\hline & 1048577 & 3764090 & 6590393 & $75.1 \%$ & 1890963 & 3455877 & $82.8 \%$ \\
\hline & 2097153 & 7523534 & 13186716 & $75.3 \%$ & 3779505 & 6917921 & $83.0 \%$ \\
\hline & 4194305 & 15052684 & 26342784 & $75.0 \%$ & 7561213 & 13826436 & $82.9 \%$ \\
\hline & 8388609 & 30111238 & 52697650 & $75.0 \%$ & 15127398 & 27660704 & $82.9 \%$ \\
\hline & 16777217 & 60193979 & 58728907 & $-2.4 \%$ & 30237773 & 30997195 & $2.5 \%$ \\
\hline Average & & & & $68.32 \%$ & & & $76.11 \%$ \\
\hline
\end{tabular}

Table 2. Results for no cycle graphs PNC01

\begin{tabular}{|c||c|c|c|c||c|c|c|}
\hline & \#Vertices & MGORC & GORC & Improve & MBFCT & BFCT & Improve \\
\hline PNC01 & 8193 & 0 & 141440 & INF & 0 & 101024 & INF \\
\hline & 16385 & 0 & 282502 & INF & 0 & 206452 & INF \\
\hline & 32769 & 0 & 577308 & INF & 0 & 421004 & INF \\
\hline & 65537 & 0 & 1175657 & INF & 0 & 840400 & INF \\
\hline & 131073 & 0 & 2326792 & INF & 0 & 1697588 & INF \\
\hline & 262145 & 0 & 4662634 & INF & 0 & 3378583 & INF \\
\hline & 524289 & 0 & 9360087 & INF & 0 & 6776352 & INF \\
\hline & 1048577 & 0 & 18799475 & INF & 0 & 13545258 & INF \\
\hline & 2097153 & 0 & 37359269 & INF & 0 & 27079159 & INF \\
\hline & 4194305 & 0 & 74824647 & INF & 0 & 54191807 & INF \\
\hline & 8388609 & 0 & 149660040 & INF & 0 & 108381976 & INF \\
\hline Average & & & & INF & & & INF \\
\hline
\end{tabular}

Table 3. Results for no cycle graphs SQNC01

\begin{tabular}{|c||c|c|c|c||c|c|c|}
\hline & \#Vertices & MGORC & GORC & Improve & MBFCT & BFCT & Improve \\
\hline SQNC01 & 4097 & 13503 & 29080 & $115.4 \%$ & 6933 & 12259 & $76.8 \%$ \\
\hline & 16385 & 51932 & 114828 & $121.1 \%$ & 26775 & 48554 & $81.3 \%$ \\
\hline & 65537 & 203158 & 479398 & $136.0 \%$ & 104971 & 191840 & $82.8 \%$ \\
\hline & 262145 & 807586 & 1961943 & $142.9 \%$ & 418223 & 733359 & $75.4 \%$ \\
\hline & 1048577 & 3211258 & 7930611 & $147.0 \%$ & 1663635 & 2919808 & $75.5 \%$ \\
\hline & 4194305 & 12817258 & 32338613 & $152.3 \%$ & 6643025 & 11873024 & $78.7 \%$ \\
\hline & 16777217 & 51213266 & 140101492 & $173.6 \%$ & 26550354 & 48035413 & $80.9 \%$ \\
\hline Average & & & & $141.19 \%$ & & & $78.77 \%$ \\
\hline
\end{tabular}


Table 4. Results for a few short cycle graphs LNC02

\begin{tabular}{|c||c|c|c|c||c|c|c|}
\hline & \#Vertices & MGORC & GORC & Improve & MBFCT & BFCT & Improve \\
\hline LNC02 & 8193 & 6442 & 11480 & $78.2 \%$ & 3240 & 5825 & $79.8 \%$ \\
\hline & 16385 & 19433 & 34692 & $78.5 \%$ & 9716 & 17916 & $84.4 \%$ \\
\hline & 32769 & 42381 & 46765 & $10.3 \%$ & 21241 & 24144 & $13.7 \%$ \\
\hline & 65537 & 90512 & 114548 & $26.6 \%$ & 45277 & 61044 & $34.8 \%$ \\
\hline & 131073 & 151996 & 267506 & $76.0 \%$ & 75979 & 141937 & $86.8 \%$ \\
\hline & 262145 & 247744 & 438869 & $77.1 \%$ & 123912 & 231623 & $86.9 \%$ \\
\hline & 524289 & 696032 & 1163851 & $67.2 \%$ & 348365 & 616440 & $77.0 \%$ \\
\hline & 1048577 & 1563907 & 1669857 & $6.8 \%$ & 782080 & 883473 & $13.0 \%$ \\
\hline & 2097153 & 1845286 & 3252535 & $76.3 \%$ & 923584 & 1729789 & $87.3 \%$ \\
\hline & 4194305 & 6011875 & 6697666 & $11.4 \%$ & 3007849 & 3560722 & $18.4 \%$ \\
\hline & 8388609 & 13820476 & 24466542 & $77.0 \%$ & 6911521 & 12969000 & $87.6 \%$ \\
\hline & 16777217 & 22339356 & 37514660 & $67.9 \%$ & 11172724 & 19902492 & $78.1 \%$ \\
\hline Average & & & & $54.44 \%$ & & & $62.32 \%$ \\
\hline
\end{tabular}

Table 5. Results for a few short cycle graphs PNC02

\begin{tabular}{|c||c|c|c|c||c|c|c|}
\hline & \#Vertices & MGORC & GORC & Improve & MBFCT & BFCT & Improve \\
\hline PNC02 & 8193 & 47185 & 59183 & $25.4 \%$ & 29484 & 30705 & $4.1 \%$ \\
\hline & 16385 & 86450 & 64450 & $-25.4 \%$ & 53720 & 54659 & $1.7 \%$ \\
\hline & 32769 & 225235 & 205400 & $-8.8 \%$ & 140052 & 142698 & $1.9 \%$ \\
\hline & 65537 & 287805 & 203174 & $-29.4 \%$ & 178108 & 180636 & $1.4 \%$ \\
\hline & 131073 & 350055 & 294782 & $-15.8 \%$ & 218465 & 212419 & $-2.8 \%$ \\
\hline & 262145 & 503878 & 386424 & $-23.3 \%$ & 314458 & 330199 & $5.0 \%$ \\
\hline & 524289 & 1316216 & 1095546 & $-16.8 \%$ & 820598 & 874808 & $6.6 \%$ \\
\hline & 1048577 & 4790290 & 3915894 & $-18.3 \%$ & 2983431 & 3175931 & $6.5 \%$ \\
\hline & 2097153 & 10428337 & 8593237 & $-17.6 \%$ & 6495202 & 6953796 & $7.1 \%$ \\
\hline & 4194305 & 23124100 & 19013193 & $-17.8 \%$ & 14408767 & 15390086 & $6.8 \%$ \\
\hline & 8388609 & 67316841 & 55413705 & $-17.7 \%$ & 41913396 & 44775974 & $6.8 \%$ \\
\hline Average & & & & $-15.05 \%$ & & & $4.10 \%$ \\
\hline
\end{tabular}

Table 6. Results for a few short cycle graphs SQNC02

\begin{tabular}{|c||c|c|c|c||c|c|c|}
\hline & \#Vertices & MGORC & GORC & Improve & MBFCT & BFCT & Improve \\
\hline SQNC02 & 4097 & 13503 & 29080 & $115.4 \%$ & 6933 & 12259 & $76.8 \%$ \\
\hline & 16385 & 51932 & 114828 & $121.1 \%$ & 26775 & 48554 & $81.3 \%$ \\
\hline & 65537 & 203158 & 479398 & $136.0 \%$ & 104971 & 191840 & $82.8 \%$ \\
\hline & 262145 & 807586 & 1961943 & $142.9 \%$ & 418223 & 733359 & $75.4 \%$ \\
\hline & 1048577 & 3211258 & 7930611 & $147.0 \%$ & 1663635 & 2919808 & $75.5 \%$ \\
\hline & 4194305 & 12817258 & 32338613 & $152.3 \%$ & 6643025 & 11873024 & $78.7 \%$ \\
\hline & 16777217 & 51213266 & 140101492 & $173.6 \%$ & 26550354 & 48035413 & $80.9 \%$ \\
\hline Average & & & & $141.19 \%$ & & & $78.77 \%$ \\
\hline
\end{tabular}


Table 7. Results for many short cycles graphs LNC03

\begin{tabular}{|c||c|c|c|c||c|c|c|}
\hline & \#Vertices & MGORC & GORC & Improve & MBFCT & BFCT & Improve \\
\hline LNC03 & 8193 & 343 & 396 & $15.3 \%$ & 173 & 196 & $13.2 \%$ \\
\hline & 16385 & 71 & 136 & $93.2 \%$ & 31 & 47 & $48.4 \%$ \\
\hline & 32769 & 112 & 275 & $145.9 \%$ & 54 & 72 & $33.1 \%$ \\
\hline & 65537 & 208 & 262 & $26.2 \%$ & 106 & 97 & $-8.5 \%$ \\
\hline & 131073 & 180 & 221 & $22.3 \%$ & 92 & 91 & $-0.7 \%$ \\
\hline & 262145 & 138 & 297 & $115.5 \%$ & 72 & 82 & $14.2 \%$ \\
\hline & 524289 & 240 & 349 & $45.4 \%$ & 120 & 110 & $-8.0 \%$ \\
\hline & 1048577 & 198 & 224 & $13.4 \%$ & 97 & 69 & $-28.7 \%$ \\
\hline & 2097153 & 149 & 313 & $110.3 \%$ & 74 & 73 & $-1.1 \%$ \\
\hline & 4194305 & 172 & 344 & $99.7 \%$ & 86 & 86 & $-0.2 \%$ \\
\hline & 8388609 & 216 & 261 & $20.9 \%$ & 106 & 102 & $-3.6 \%$ \\
\hline & 16777217 & 193 & 202 & $4.6 \%$ & 95 & 100 & $5.7 \%$ \\
\hline Average & & & & $59.39 \%$ & & & $5.32 \%$ \\
\hline
\end{tabular}

Table 8. Results for many short cycles graphs PNC03

\begin{tabular}{|c||c|c|c|c||c|c|c|}
\hline & \#Vertices & MGORC & GORC & Improve & MBFCT & BFCT & Improve \\
\hline PNC03 & 8193 & 461 & 2713 & $489.1 \%$ & 327 & 865 & $164.6 \%$ \\
\hline & 16385 & 473 & 3046 & $543.5 \%$ & 256 & 451 & $75.7 \%$ \\
\hline & 32769 & 468 & 3843 & $721.2 \%$ & 248 & 513 & $106.9 \%$ \\
\hline & 65537 & 341 & 3030 & $788.0 \%$ & 165 & 829 & $402.9 \%$ \\
\hline & 131073 & 967 & 3842 & $297.1 \%$ & 697 & 686 & $-1.7 \%$ \\
\hline & 262145 & 381 & 3670 & $863.8 \%$ & 214 & 339 & $58.7 \%$ \\
\hline & 524289 & 1144 & 4174 & $265.0 \%$ & 620 & 525 & $-15.3 \%$ \\
\hline & 1048577 & 177 & 2743 & $1453.5 \%$ & 113 & 244 & $116.9 \%$ \\
\hline & 2097153 & 1125 & 3747 & $233.0 \%$ & 617 & 552 & $-10.6 \%$ \\
\hline & 4194305 & 314 & 2063 & $557.5 \%$ & 175 & 394 & $124.9 \%$ \\
\hline & 8388609 & 632 & 2628 & $315.5 \%$ & 292 & 409 & $40.1 \%$ \\
\hline Average & & & & $593.38 \%$ & & & $96.65 \%$ \\
\hline
\end{tabular}

Table 9. Results for many short cycles graphs SQNC03

\begin{tabular}{|c||c|c|c|c||c|c|c|}
\hline & \#Vertices & MGORC & GORC & Improve & MBFCT & BFCT & Improve \\
\hline SQNC03 & 4097 & 109 & 487 & $345.3 \%$ & 55 & 173 & $216.1 \%$ \\
\hline & 16385 & 74 & 584 & $686.5 \%$ & 34 & 249 & $625.0 \%$ \\
\hline & 65537 & 250 & 1101 & $340.5 \%$ & 130 & 493 & $280.1 \%$ \\
\hline & 262145 & 477 & 3779 & $691.6 \%$ & 243 & 1077 & $343.2 \%$ \\
\hline & 1048577 & 1818 & 7839 & $331.1 \%$ & 950 & 2441 & $157.0 \%$ \\
\hline & 4194305 & 2934 & 7534 & $156.8 \%$ & 1536 & 4593 & $199.0 \%$ \\
\hline & 16777217 & 5896 & 28282 & $379.7 \%$ & 3066 & 8719 & $184.4 \%$ \\
\hline Average & & & & $418.79 \%$ & & & $286.40 \%$ \\
\hline
\end{tabular}


Table 10. Results for a few long cycles graphs LNC04

\begin{tabular}{|c||c|c|c|c||c|c|c|}
\hline & \#Vertices & MGORC & GORC & Improve & MBFCT & BFCT & Improve \\
\hline LNC04 & 8193 & 26711 & 54962 & $105.8 \%$ & 12910 & 20687 & $60.2 \%$ \\
\hline & 16385 & 92773 & 148990 & $60.6 \%$ & 41107 & 56489 & $37.4 \%$ \\
\hline & 32769 & 251488 & 349870 & $39.1 \%$ & 122379 & 145197 & $18.6 \%$ \\
\hline & 65537 & 881848 & 948447 & $7.6 \%$ & 365853 & 376227 & $2.8 \%$ \\
\hline & 131073 & 1980993 & 2097090 & $5.9 \%$ & 874778 & 898293 & $2.7 \%$ \\
\hline & 262145 & 4467623 & 5460818 & $22.2 \%$ & 1892207 & 2215706 & $17.1 \%$ \\
\hline & 524289 & 11883080 & 11385873 & $-4.2 \%$ & 4968220 & 5017279 & $1.0 \%$ \\
\hline & 1048577 & 25741146 & 28462486 & $10.6 \%$ & 10792331 & 12567955 & $16.5 \%$ \\
\hline & 2097153 & 62102224 & 61088246 & $-1.6 \%$ & 26089374 & 27892978 & $6.9 \%$ \\
\hline & 4194305 & 121983730 & 128064383 & $5.0 \%$ & 53771346 & 59798591 & $11.2 \%$ \\
\hline & 8388609 & 275905623 & 286903304 & $4.0 \%$ & 112925426 & 135532732 & $20.0 \%$ \\
\hline & 16777217 & 538729562 & 664472322 & $23.3 \%$ & 231721552 & 299999324 & $29.5 \%$ \\
\hline Average & & & \multicolumn{1}{c|}{$23.19 \%$} & & & $18.66 \%$ \\
\hline
\end{tabular}

Table 11. Results for a few long cycles graphs PNC04

\begin{tabular}{|c||c|c|c|c||c|c|c|}
\hline & \#Vertices & MGORC & GORC & Improve & MBFCT & BFCT & Improve \\
\hline PNC04 & 8193 & 96459 & 82610 & $-14.4 \%$ & 55914 & 37737 & $-32.5 \%$ \\
\hline & 16385 & 363660 & 189675 & $-47.8 \%$ & 222163 & 89015 & $-59.9 \%$ \\
\hline & 32769 & 1032742 & 416393 & $-59.7 \%$ & 633831 & 215134 & $-66.1 \%$ \\
\hline & 65537 & 2475918 & 932459 & $-62.3 \%$ & 1574038 & 485710 & $-69.1 \%$ \\
\hline & 131073 & 3959249 & 2244526 & $-43.3 \%$ & 2482065 & 1042791 & $-58.0 \%$ \\
\hline & 262145 & 11673063 & 4881046 & $-58.2 \%$ & 6672867 & 2282332 & $-65.8 \%$ \\
\hline & 524289 & 26999818 & 10280214 & $-61.9 \%$ & 16888696 & 4707412 & $-72.1 \%$ \\
\hline & 1048577 & 56517150 & 21103100 & $-62.7 \%$ & 35114982 & 9594361 & $-72.7 \%$ \\
\hline & 2097153 & 118365443 & 44260038 & $-62.6 \%$ & 74302031 & 20881883 & $-71.9 \%$ \\
\hline & 4194305 & 225343295 & 95936613 & $-57.4 \%$ & 147809535 & 43256228 & $-70.7 \%$ \\
\hline & 8388609 & 517666048 & 189688611 & $-63.4 \%$ & 328137483 & 88085199 & $-73.2 \%$ \\
\hline Average & & & & $-53.97 \%$ & & & $-63.74 \%$ \\
\hline
\end{tabular}

Table 12. Results for a few long cycles graphs SQNC04

\begin{tabular}{|c||c|c|c|c||c|c|c|}
\hline & \#Vertices & MGORC & GORC & Improve & MBFCT & BFCT & Improve \\
\hline SQNC04 & 4097 & 32707 & 30880 & $-5.6 \%$ & 15213 & 13944 & $-8.3 \%$ \\
\hline & 16385 & 184893 & 170162 & $-8.0 \%$ & 81463 & 67445 & $-17.2 \%$ \\
\hline & 65538 & 573055 & 746841 & $30.3 \%$ & 254273 & 297501 & $17.0 \%$ \\
\hline & 262145 & 1313928 & 3264595 & $148.5 \%$ & 560271 & 1389863 & $148.1 \%$ \\
\hline & 1048577 & 2338506 & 15068659 & $544.4 \%$ & 1099832 & 6288913 & $471.8 \%$ \\
\hline & 4194305 & 6204248 & 68242481 & $999.9 \%$ & 2710118 & 27430729 & $912.2 \%$ \\
\hline & 16777217 & 14024018 & 272653950 & $1844.2 \%$ & 6681120 & 121993247 & $1725.9 \%$ \\
\hline Average & & & & $507.67 \%$ & & & $464.21 \%$ \\
\hline
\end{tabular}


Table 13. Results for Hamiltonian cycle graphs LNC05

\begin{tabular}{|c||c|c|c|c||c|c|c|}
\hline & \#Vertices & MGORC & GORC & Improve & MBFCT & BFCT & Improve \\
\hline LNC05 & 8193 & 163438 & 172029 & $5.3 \%$ & 98947 & 91658 & $-7.4 \%$ \\
\hline & 16385 & 346868 & 364541 & $5.1 \%$ & 215632 & 202262 & $-6.2 \%$ \\
\hline & 32769 & 753945 & 803979 & $6.6 \%$ & 458880 & 436855 & $-4.8 \%$ \\
\hline & 65537 & 1633918 & 1733490 & $6.1 \%$ & 999207 & 934728 & $-6.5 \%$ \\
\hline & 131073 & 3499646 & 3789556 & $8.3 \%$ & 2082565 & 2027643 & $-2.6 \%$ \\
\hline & 262145 & 7599126 & 7828071 & $3.0 \%$ & 4637622 & 4329335 & $-6.6 \%$ \\
\hline & 524289 & 15608355 & 16876480 & $8.1 \%$ & 9456863 & 9179454 & $-2.9 \%$ \\
\hline & 1048577 & 33572684 & 35232411 & $4.9 \%$ & 20445301 & 19287842 & $-5.7 \%$ \\
\hline & 2097153 & 69453620 & 74266615 & $6.9 \%$ & 42213747 & 40477734 & $-4.1 \%$ \\
\hline & 4194305 & 144322754 & 156777150 & $8.6 \%$ & 89613042 & 84456141 & $-5.8 \%$ \\
\hline & 8388609 & 314273826 & 331798694 & $5.6 \%$ & 189346386 & 181584841 & $-4.1 \%$ \\
\hline & 16777217 & 637684719 & 668256739 & $4.8 \%$ & 387236035 & 364980712 & $-5.7 \%$ \\
\hline Average & & & & $6.11 \%$ & & & $-5.20 \%$ \\
\hline
\end{tabular}

Table 14. Results for Hamiltonian cycle graphs PNC05

\begin{tabular}{|c||c|c|c|c||c|c|c|}
\hline & \#Vertices & MGORC & GORC & Improve & MBFCT & BFCT & Improve \\
\hline PNC05 & 8194 & 82594 & 90718 & $9.8 \%$ & 54836 & 47319 & $-13.7 \%$ \\
\hline & 16386 & 179350 & 203306 & $13.4 \%$ & 116631 & 100007 & $-14.3 \%$ \\
\hline & 32770 & 361404 & 401511 & $11.1 \%$ & 249244 & 210670 & $-15.5 \%$ \\
\hline & 65538 & 785227 & 870523 & $10.9 \%$ & 516925 & 443592 & $-14.2 \%$ \\
\hline & 131074 & 1732688 & 1680790 & $-3.0 \%$ & 1096942 & 901936 & $-17.8 \%$ \\
\hline & 262146 & 3462811 & 3965235 & $14.5 \%$ & 2232233 & 1928211 & $-13.6 \%$ \\
\hline & 524290 & 7714697 & 7216528 & $-6.5 \%$ & 4579138 & 4039317 & $-11.8 \%$ \\
\hline & 1048578 & 16872712 & 16794782 & $-0.5 \%$ & 9786195 & 8450277 & $-13.7 \%$ \\
\hline & 2097154 & 32924136 & 37489022 & $13.9 \%$ & 19973817 & 17243048 & $-13.7 \%$ \\
\hline & 4194306 & 63798970 & 71903161 & $12.7 \%$ & 41054033 & 35357906 & $-13.9 \%$ \\
\hline & 8388610 & 154010041 & 151672136 & $-1.5 \%$ & 86223637 & 74532047 & $-13.6 \%$ \\
\hline Average & & & & $-1.50 \%$ & & & $-14.16 \%$ \\
\hline
\end{tabular}

Table 15. Results for Hamiltonian cycle graphs SQNC05

\begin{tabular}{|c||c|c|c|c||c|c|c|}
\hline & \#Vertices & MGORC & GORC & Improve & MBFCT & BFCT & Improve \\
\hline SQNC05 & 4098 & 70976 & 79742 & $12.4 \%$ & 44744 & 41610 & $-7.0 \%$ \\
\hline & 16386 & 360423 & 365960 & $1.5 \%$ & 207067 & 197097 & $-4.8 \%$ \\
\hline & 65538 & 1644198 & 1712316 & $4.1 \%$ & 983910 & 928755 & $-5.6 \%$ \\
\hline & 262146 & 7650803 & 8070454 & $5.5 \%$ & 4679524 & 4297402 & $-8.2 \%$ \\
\hline & 1048578 & 32523850 & 34451433 & $5.9 \%$ & 19818466 & 18737042 & $-5.5 \%$ \\
\hline & 4194306 & 151666794 & 149113446 & $-1.7 \%$ & 91909813 & 82151343 & $-10.6 \%$ \\
\hline & 16777218 & 648487235 & 648211387 & $0.0 \%$ & 394621759 & 353276864 & $-10.5 \%$ \\
\hline Average & & & & $3.96 \%$ & & & $-7.46 \%$ \\
\hline
\end{tabular}


Table 16. Results for random graphs RAND-5

\begin{tabular}{|c||c|c|c||c|c|c|}
\hline Negative Weight & MGORC & GORC & Improve & MBFCT & BFCT & Improve \\
\hline 0 & 0 & 7780968 & INF & 0 & 4578450 & INF \\
\hline-1000 & 1133358 & 8892373 & $684.6 \%$ & 341958 & 5350722 & $1464.7 \%$ \\
\hline-2000 & 2080317 & 9588453 & $360.9 \%$ & 762572 & 6481434 & $749.9 \%$ \\
\hline-4000 & 260253 & 3665786 & $1308.5 \%$ & 211730 & 5139325 & $2327.3 \%$ \\
\hline-6000 & 328345 & 884558 & $169.4 \%$ & 218531 & 1634924 & $648.1 \%$ \\
\hline-8000 & 224668 & 41588 & $-81.5 \%$ & 115428 & 721280 & $524.9 \%$ \\
\hline-16000 & 603 & 541 & $-10.4 \%$ & 328455 & 237747 & $-27.6 \%$ \\
\hline-32000 & 175 & 174 & $-0.5 \%$ & 329102 & 143538 & $-56.4 \%$ \\
\hline-64000 & 51 & 48 & $-4.3 \%$ & 380534 & 55175 & $-85.5 \%$ \\
\hline
\end{tabular}

Table 17. General results for GORC

Table 18. General results for BFCT

\begin{tabular}{|c||c|c|}
\hline & Improve & Degrade \\
\hline $100 \%<X$ & $36.1 \%$ & $0.0 \%$ \\
\hline $10 \%<X \leq 100 \%$ & $27.8 \%$ & $17.0 \%$ \\
\hline$X \leq 10 \%$ & $12.8 \%$ & $6.3 \%$ \\
\hline \hline Total & $76.7 \%$ & $23.3 \%$ \\
\hline
\end{tabular}

\begin{tabular}{|c||c|c|}
\hline & Improve & Degrade \\
\hline $100 \%<X$ & $24.8 \%$ & $0.0 \%$ \\
\hline $10 \%<X \leq 100 \%$ & $29.3 \%$ & $25.7 \%$ \\
\hline$X \leq 10 \%$ & $9.6 \%$ & $10.6 \%$ \\
\hline \hline Total & $63.7 \%$ & $36.3 \%$ \\
\hline
\end{tabular}

\section{Appendix II}

\section{Proof of Lemma 1}

Consider a negative cost cycle $C$ in a directed graph $G(V, E)$ where $C=\left\{v_{0}, v_{1}\right.$, $\left.\ldots, v_{n-1}\right\}$. The accumulated costs on $C$ starting from $v_{0}$ are $\left\{C_{1,0}, C_{1,1}, \ldots\right.$, $\left.C_{1, n-1}\right\}$, where $C_{1, k}$ is the accumulated cost at vertex $v_{k}$ starting from $v_{0}$.

First of all, at the end of the cycle, i.e. $v_{n-1}$, the accumulated cost $\left(W_{\text {cycle }}\right)$ along this cycle must be negative. Secondly, there is at least one maximum accumulated cost $C_{\max }$ on this cycle. Finally, there must be at least one edge, $\left(v_{i}, v_{j}\right)$, where $j=i+1$, that is negative since there must be at least one negative edge on a negative cycle. Note that we can change the ordering of the vertices by choosing another "starting vertex". Suppose that we take $v_{k}$ as the starting vertex where $\mathrm{v}_{k}$ has the largest accumulated cost (chose the last one if there is more than one), we will have the accumulated costs $\left\{C_{2, k+1}, C_{2, k+2}, \ldots\right.$, $\left.C_{2, n-1}, C_{2,0}, C_{2,1}, \ldots, C_{2, k}\right\}$.

Now we will have the path $\left\{v_{k+1}, v_{k+2}, \ldots, v_{n-1}, v_{0}, v_{1}, \ldots, v_{k}\right\}$. Then we will have two sets of vertices. The first set contains the vertices in $\left\{v_{k+1}, v_{k+2}\right.$, $\left.\ldots, v_{n-1}\right\}$. For any vertex $v$ in this set, $C_{2}, v=C_{1}, v-C_{\max }<0$, where $C_{\text {max }} \geqq 0$ because otherwise, $v_{0}$ is already the correct starting point. The second set contains the vertices in $\left\{v_{0}, v_{1}, \ldots, v_{k}\right\}$. For any vertex $v$ in this set, $C_{2}, v=$ $C_{1}, v+W_{\text {cycle }}-C_{\max }<0$. 